\title{
8. Cholesterol transporters in lactating and nonlactating human mammary tissue
}

\author{
C. Albrecht, X. Huang and E.C. Ontsouka \\ Institute of Biochemistry and Molecular Medicine, Faculty of Medicine, University of Bern, \\ Buehlstrasse 28, 3012 Bern, Switzerland; christiane.albrecht@ibmm.unibe.ch
}

\section{Abstract}

In mammals milk is the principal nutrient for neonates at birth. The basic milk composition is similar between different mammals, but the content of individual constituents such as lipids may differ significantly from one species to another. The milk fat fraction is mainly composed of triglycerides which account for more than 95\% of the lipids found in human and bovine milk. Though sterols and in particular cholesterol, the predominant milk sterol, represent less than $0.5 \%$ of the total milk lipid fraction, they are of ultimate importance for biological processes such as the formation of biological membranes or as precursors for steroid hormone synthesis. Cholesterol found in milk originates either from blood uptake or from local synthesis. This chapter provides an overview of cholesterol exchanges between the blood, the mammary tissue and the milk. The current knowledge on the expression, localization and function of candidate cholesterol transporters in mammary tissues of human, murine and bovine origin is summarized. Different mechanisms of how cholesterol can be transferred via the mammary tissue into milk, and which active cholesterol transporters are likely to play a role in this process will be discussed.

Keywords: cholesterol transport, milk composition, ABC transporter, mammary gland 


\section{Key facts}

- Cholesterol is a crucial molecule which, as a cell membrane constituent, is essential for cell growth and renewal as well as for the synthesis of specific hormones.

- Milk and its derived products are one of the most important sources of cholesterol in the human diet (both adults and infants). Regarding its role as a nutrient found in milk considerable and often controversial effects of cholesterol on human health are discussed. In this context it seems important to distinguish cholesterol effects in babies/infants as opposed to adults.

- Babies consume very high amounts of cholesterol per $\mathrm{kg}$ of body weight compared to adults without showing detrimental effects attributed to high cholesterol intake. Interestingly, the increase of serum cholesterol levels in breastfed babies is more pronounced as compared to milk formula fed babies.

- The identification of active cholesterol transporters in other peripheral tissues have revolutionized the current thinking of how cholesterol is transported or exported from epithelial cells. Therefore, the current concept of cholesterol trafficking in the mammary gland needs re-thinking and the role of active cholesterol transporters in this process has to be elucidated.

\section{Summary points}

- Cholesterol is the predominant sterol found in milk. It enters the latter from the blood where it circulates bound to various carrier proteins such as very low density lipoprotein, low density lipoprotein, and high density lipoprotein.

- The transcellular transport of cholesterol in the mammary gland implies an uptake from the blood circulation, redistribution in cytosolic compartments and release into milk.

- The cholesterol transport into milk involves energy-dependent and energy-independent processes that operate at the basal side of the blood-milk barrier (facing the blood, cholesterol uptake) and at the opposite apical side (facing the milk, cholesterol release).

- The transport of cholesterol is likely to be mediated by universal membrane located cholesterol transporters (e.g. $A B C A 1, A B C G 1$ ), but also by passive diffusion and by MFG secretion, a specific secretion mode inherent to mammary epithelial cells.

- mRNA and protein expression of $A B C$ cholesterol transporters was found in the mammary epithelium of the blood-milk barrier in lactating and nonlactating mammary tissue of diverse mammals including humans.

- The mechanisms and regulatory pathways mediating cholesterol transport in the mammary gland and their functional relevance in terms of cholesterol release into milk needs further complementary investigations. 


\section{Abbreviations}

$\begin{array}{ll}\text { ABC } & \text { ATP-binding cassette } \\ \text { apo-A1 } & \text { Apolipoprotein-A1 } \\ \text { ECD } & \text { Extracellular domain } \\ \text { HDL } & \text { High density lipoprotein } \\ \text { LDL } & \text { Low density lipoprotein } \\ \text { MFG } & \text { Milk fat globule } \\ \text { NBD } & \text { Nucleotide binding domain } \\ \text { NPC } & \text { Niemann-Pick type C } \\ \text { NPC1L1 } & \text { Niemann-Pick type C1 like 1 } \\ \text { TMD } & \text { Transmembrane domain } \\ \text { VLDL } & \text { Very low density lipoprotein }\end{array}$

\subsection{Introduction}

\subsubsection{Cholesterol in blood and milk}

Throughout lactation mammary alveolar epithelial cells synthesize, store, and release specific milk constituents, including lipids, into the alveolar milk. Cholesterol found in circulating blood originates either from the liver, where acetyl-coenzyme A is used as a precursor for cholesterol biosynthesis, or from the dietary uptake in the intestine. The total cholesterol concentration measured in the bloodstream consists of fractions bound to different carrier lipoproteins such as VLDL, LDL, HDL and chylomicrons, which are synthesized and released into the blood by the liver and the intestine.

The total blood serum cholesterol level measured in newborn babies' blood cord is approx. 70.3 $\mathrm{mg} / \mathrm{dl}$ and this level increases with both breast-milk and milk formula intake (Mishkel, 1974). In mice, the total blood serum cholesterol level slightly varies from $170 \mathrm{mg} / \mathrm{dl}$ at day three of birth to $200 \mathrm{mg} / \mathrm{dl}$ later on (Wang et al., 2011), whereas in cow blood serum cholesterol levels are between 96.5 and $270.2 \mathrm{mg} / \mathrm{dl}$ (Mani et al., 2009). In adult humans, the total blood serum cholesterol level varies between 204.6 and $297.2 \mathrm{mg} / \mathrm{dl}$. These values characterize the transition from healthy $(\leq 204 \mathrm{mg} / \mathrm{dl})$ to disease conditions. Factors such as weight gain during the pregnancy and the number of pregnancies might affect the blood and the milk lipid profiles in healthy women.

The cholesterol requirements of the mammary tissue increase with the onset of lactation. An increase of blood and milk cholesterol levels after parturition has been found to be concomitant with the up-regulation of cholesterol biosynthetic genes in the liver (Viturro et al., 2009), confirming that hepatic cholesterol is required to cover the increasing mammary tissue demand due to milk synthesis. Figure 8.1 gives an overview of cholesterol exchanges between the blood, the mammary tissue and the milk with its uptake occurring at the basolateral membrane of the secretory cells facing the bloodstream, and cholesterol release into milk taking place at the apical 


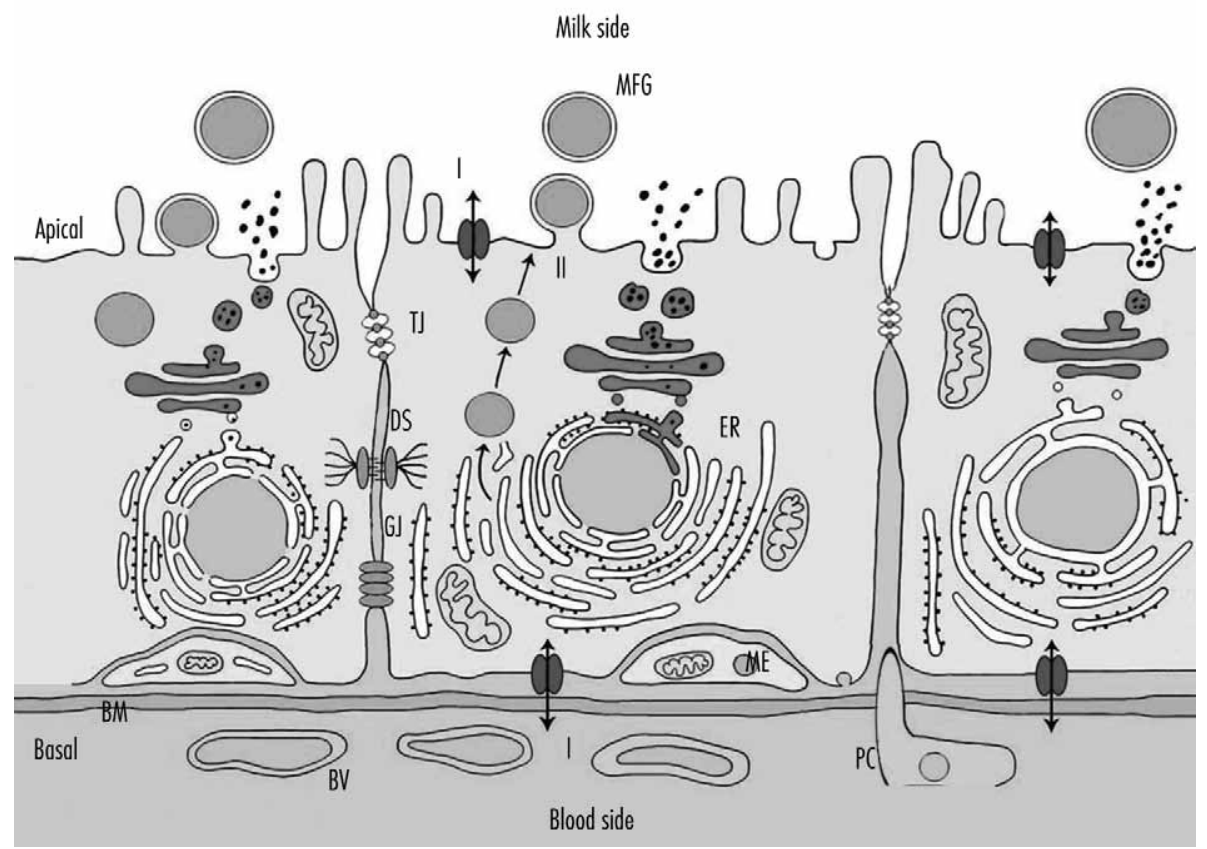

Figure 8.1. Milk secretion pathways relevant for lipids and cholesterol in mammary alveolar epithelial cells. Pathway I shows transporter proteins responsible for the direct movement of ions, water, glucose, drugs, xenobiotics and possibly lipids/cholesterol across both apical and basal plasma membranes. Potentially involved proteins of pathway I in the context of lipid and cholesterol transfer include scavenger receptors at the basal membrane and $A B C$ transporter proteins at both the basal and the apical side of the plasma membrane. Mechanism II illustrates the milk fat and lipid secretion pathway with formation of lipid droplets that move towards the apical membrane for release as membrane bound milk fat globules (MFG). Other mechanisms such as exocytotic secretion, vesicular transcytosis and paracellular pathways currently seem to be less relevant for lipid/cholesterol transport and are therefore not shown. ER: endoplasmic reticulum, BM: basement membrane, PC: plasma cell, TJ: tight junction, GJ: gap junction, DS: desmosome, ME: myoepithelial cell, BV: blood vessel.

side of the secretory cells. The blood-borne carrier lipoproteins such as LDL are the principal cholesterol delivery proteins to diverse peripheral extra-hepatic tissues and organs including the mammary tissue (Monks et al., 2001). Furthermore, the presence of the LDL receptor in mouse mammary tissues, but also an LDL receptor- independent LDL uptake has been demonstrated (Monks et al., 2001).

Cholesterol is a component of the milk lipid fraction. The milk lipids exist as globules emulsified in the aqueous phase and are the major source of energy for neonates. They form the third major milk constituent beside water and lactose (Table 8.1). A wide variability in the milk lipid content as well as in the cholesterol content of milk derived products is described across various mammalian species (Table 8.2). The milk lipid content increases during feeding and varies over the diurnal cycle (Jensen, 1996). Moreover, the milk lipid fraction and its individual components such as cholesterol undergo changes during the course of lactation (Jensen, 1996). In humans, 
Table 8.1. Constituents in human milk. ${ }^{1}$

\begin{tabular}{lcc}
\hline Milk constituent & Colostrum & Mature milk \\
Lactose (g/l) & $20-30$ & 67 \\
Oligosaccharides (g/l) & $22-24$ & $12-14$ \\
Total protein (g/l) & 16 & 9 \\
Total lipids (\%) & 2 & 3.5 \\
Triglycerides (\% total lipids) & $97-98$ & $97-98$ \\
Cholesterol (\% total lipids) & $0.7-1.3$ & $0.4-0.5$ \\
Cholesterol (mg/dl) & $>30$ & $10-20$ \\
Phospholipids (\% total lipids) & 1.1 & $0.6-0.8$ \\
Fatty acids (weight \%) & 88 & 88 \\
\hline
\end{tabular}

1 This table has been adapted from Jensen (1996) and Picciano (2001) with permission from Elsevier. In the original references additional information for other milk constituents (e.g. vitamins and minerals) can be found. Data are expressed as range or single values.

Table 8.2. Total lipid and cholesterol levels in milk commonly consumed by humans. ${ }^{1}$

\begin{tabular}{lll}
\hline Milk origin & Total lipids (\%) & Cholesterol (mg per 100 g fat) \\
Cow & $1.38-5.10$ & $239-300$ \\
Sheep & $5.79-6.45$ & $288 \pm 42$ \\
Goat & $2.75-6.43$ & $341 \pm 15$ \\
\hline
\end{tabular}

${ }^{1}$ Data are adapted from Jandal (1996), Jensen (2002) and Park et al. (2007) and are published with permission from Elsevier.

the milk lipid content is low in the colostrum ( 2\%) and increases in mature milk (3-4.8\%) (Table 8.1). In cow milk an inverse milk lipid profile has been shown, where milk lipids were higher immediately after parturition (i.e. in colostrum) than later in mature milk (Ontsouka et al., 2003). Cholesterol is the predominant component of the milk sterols (95\% of total sterols). The cholesterol level in human milk is elevated in the colostrum and decreases to approx. 10-20 $\mathrm{mg} / \mathrm{dl}$ in mature milk (Table 8.1). These levels are comparable to the cholesterol content in bovine mature milk (10-30 mg/dl) corresponding to roughly $10 \%$ of blood cholesterol levels (Jensen, 2002). Milk cholesterol exists also in the form of cholesteryl esters which account for 5-15\% of total milk cholesterol. This fraction decreases from colostrum to mature milk. In cow milk which is preferentially consumed by humans (both infants and adults) the cholesterol content is low in colostrum and increases in the mature milk. Furthermore, the blood cholesterol, and more generally the blood lipid profile, is influenced by several factors including the diet. 


\section{Albrecht, X. Huang and E.C. Ontsouka}

\subsubsection{Multifaceted health effects of cholesterol}

Cholesterol contained in milk and other dairy products is an important source of cholesterol in the human diet. Although cholesterol is a 'minor' milk lipid component $(0.5 \%$ of total milk lipids), its role in public health, particularly in the context of cardiovascular diseases, is of major interest. Cholesterol is an indispensable constituent of biological membranes that is required for cell signaling, such as the hedgehog signaling which controls the precise pattern of embryonic structure and development (Gofflot et al., 2003). It is also a precursor in the synthesis of multiple sterol-based bioactive compounds, e.g. estrogen, testosterone and vitamin D.

Recently, it has been demonstrated that in breastfed babies at six months of age the total blood serum cholesterol levels reach up to $194 \mathrm{mg} / \mathrm{dl}$, i.e. almost the same concentration as measured in the blood serum of adults. The increase of blood serum cholesterol (including its LDL fraction) is more pronounced in breastfed infants already at one month old compared to milk formula fed infants (Isomura et al., 2011). It is known that the milk intake by infants in the first six months postpartum ranges from 450-1,200 $\mathrm{ml} /$ day, and that the average human milk cholesterol content is between 100-200 mg/l (Picciano, 2001). Therefore, the daily milk cholesterol intake by infants in the early life may vary from $45-240 \mathrm{mg} /$ day. Related to the average infant body weight at six months of age, this implies a daily cholesterol intake of 6.5-34 mg cholesterol per kg body weight. Assuming an average body weight of $80 \mathrm{~kg}$ in adults this would represent the equivalent of 0.52 to $2.7 \mathrm{~g}$ cholesterol per day. Compared to the recommended daily cholesterol intake of approx. 200-400 mg, it is obvious that the cholesterol requirements for adult and newborn/infant organisms are markedly different. In contrast to adults, detrimental effects of high cholesterol consumption associated with breastfeeding have not been reported yet. Moreover, although a consensus is still missing, there are reports describing potentially beneficial effects of exclusive breastfeeding. It has been suggested that high total blood serum cholesterol found in breastfed infants in early postnatal life might prevent them from the risk of developing cardiovascular diseases in older ages (Owen et al., 2005; Rudnicka et al., 2007). On the other hand, it is reported that LDL particles may play a role in facilitating the generation of antibodies and that a reduced ability to generate antibodies was seen in infants having low blood LDL-cholesterol levels. Compared to breastfed infants, those infants with low blood cholesterol were reported to be more susceptible to infections and allergic diseases and had increased mortality (Picaud et al., 2010; Victora et al., 1987). Definitely, in early postnatal life babies need plenty of cholesterol to match the cholesterol requirements linked to rapid organ growth, development and maturation, especially of the skeletal and the nervous system.

\subsection{Mechanisms of cholesterol transport in mammary tissues}

Scientific literature describing the transport of cholesterol in lactating and nonlactating human mammary tissues is scarce. However, it is likely that the principles governing the transport of cholesterol in mammary tissues are not fundamentally different from those known in other mammalian peripheral tissues or cells. Accordingly, Figure 8.1 (mechanism I and II) and 
Figure 8.2 (pathways A-C) illustrate possible pathways of cholesterol transfer from the blood side to the apical plasma membrane of mammary alveolar epithelial cells followed by its release into milk.

Like other nutrients entering the milk, cholesterol must cross the apical plasma membrane of mammary alveolar epithelial cells to enter the alveolar space. Cholesterol taken up at the basal side can follow the transcellular pathway towards the apical membrane for its ultimate release into

Milk side

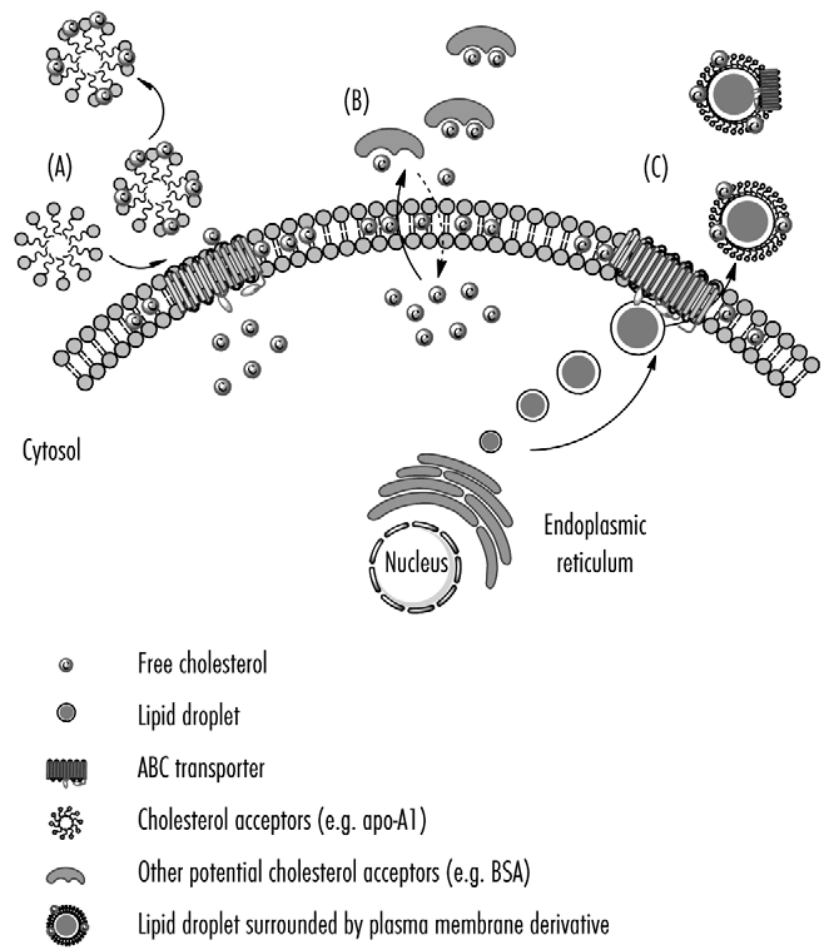

Figure 8.2. Overview of potential cholesterol transfer mechanisms at the apical plasma membrane of secretory mammary epithelial cells for release into milk. $A B C$ transporters present at the apical plasma membrane mediate the transfer of cholesterol to lipid-poor apo-A1 (ABCA1) or HDL (ABCG1) (pathway A). Pathway A requires the hydrolysis of ATP which, in the case of ABCA1 induces conformational changes that allows apolipoprotein (apo)-A1 binding and further cholesterol transfer and release. Alternatively cholesterol could cross the apical plasma membrane facing the alveolar lumen by diffusion following the concentration gradient and attach to potential cholesterol acceptors, such as bovine serum albumin (or others), in the time scale of hours (pathway B). The milk fat globule secretion mode (pathway C) involves formation of small lipid droplets in the endoplasmic reticulum that then migrate towards the apical membrane as they mature. At the apical region, lipid droplets are progressively surrounded by the plasma membrane and are then pinched off into the milk. 


\section{Albrecht, X. Huang and E.C. Ontsouka}

milk (Figure 8.1, pathway I). At the apical plasma membrane, different mechanisms are likely to operate which involve: (1) active transporters (Figure 8.1, pathway I; Figure 8.2, pathway A); (2) passive diffusion processes (Figure 8.2, pathway B); and (3) a specific secretion mode inherent to mammary epithelial cells (Figure 8.1, pathway II; Figure 8.2, pathway C). Recent findings in the field of cholesterol transport in mammary tissue support the contribution of active cholesterol transporters at both the basal and the apical side of mammary alveolar epithelial cells.

\subsubsection{Active cholesterol transport}

Active cholesterol transport involves the participation of integral plasma membrane proteins, and members of the ABC transporter family have been previously implicated in cholesterol trafficking across membranes in peripheral tissues (Lee et al., 2002; Stefulj et al., 2009). The prerequisite for an involvement of candidate $\mathrm{ABC}$ proteins in transcellular cholesterol transport in human mammary tissue is their plasma membrane localization (Figure 8.1 and 8.2). In a recent study it was demonstrated that $\mathrm{ABC}$ transporters - described in more detail thereafter - are important candidate proteins for cholesterol transfer in the mammary gland of mammals, including humans. The cholesterol transport proteins were found on both the basal and apical side of secretory mammary cells by immunostaining (Mani et al., 2010). Furthermore, the proteome of the plasma membrane covering the MFG prepared from human and cow milk, demonstrated the presence of $\mathrm{ABC}$ transporters (Reinhardt and Lippolis, 2006), supporting their potential role in cholesterol transfer into milk.

$A B C$ transporters are one of the largest families of integral plasma membrane proteins involved in the transport of various substrates across cellular membranes. The transport process requires the hydrolysis of ATP, and substrates include ions, sugars, amino acids, vitamins, lipids, and drugs as well as larger molecules, such as oligosaccharides, oligopeptides and even high molecular weight proteins. ABC transporters consists of intracellular NBD and TMD composed of 6-11 membrane-spanning $a$-helices. A functional $\mathrm{ABC}$ transporter can be either a complete protein with two NBD and two TMD (full transporter) or a dimer of two half transporters (one NBD and one TMD each). To date, seven subfamilies (named from A to G) have been identified and characterized.

Members of the subfamilies ABCA and ABCG are the most promising candidates regarding the transport of cholesterol in the mammary gland because they were identified as cholesterol transporters in other mammalian cell types such as gall-bladder epithelial cells (Lee et al., 2002) or placental endothelial cells (Stefulj et al., 2009). ABC proteins of the subfamily A differ from other ABC transporters by the presence of two large ECDs whose ATP hydrolysis-dependent conformational changes are required for cholesterol transport (Nagao et al., 2012). In humans, functional defects in ABCA transporter proteins are associated with various diseases such as HDL deficiency, pulmonary surfactant deficiency in newborns, or Stargardt disease 1 (Ueda, 2011). In the next section some of the most promising candidate cholesterol transporters in the mammary gland are briefly described. 


\section{Cholesterol transporters in human mammary tissue}

\section{$A B C A$ subfamily}

$A B C A 1$ plays a major role in the maintenance of cellular cholesterol homeostasis and HDL metabolism. ABCA1 is a full transporter of $220 \mathrm{kDa}$ which is localized mainly on the plasma membrane and some intracellular compartments like the Golgi network and recycling endosomes (Takahashi et al., 2005). The cholesterol transport mediated by ABCA1 necessitates the interaction between $\mathrm{ABCA} 1$ and lipid-poor apo-A1 which functions as its main cholesterol acceptor. In a recent study it has been demonstrated that the ATP hydrolysis at the NBD induces conformational changes in the two ECD of ABCA1 that allows apoA-1 binding and subsequent cholesterol efflux (Nagao et al., 2012). The interaction of apoA-1 with ABCA1 induces intracellular cascade events which involve various signalling proteins (Liu and Tang, 2012). Furthermore, the expression of ABCA1 is regulated at both transcriptional and posttranscriptional levels (Ueda, 2011).

$A B C A 7$, another member of the A subfamily, is a full transporter with a highest homology to ABCA1. The protein has been identified at the plasma membrane (Linsel-Nitschke et al., 2005). Although the physiological functions of ABCA7 are still unclear, its role in the process of phagocytosis in macrophages has been suggested. ABCA7 null-mice show reduced HDL levels and alterations in adipose mass. In vitro studies revealed that ABCA7 promotes the efflux of cellular phospholipids onto lipoproteins (Wang et al., 2003).

\section{$A B C G$ subfamily}

$A B C G 1$ has structural and expression similarities to ABCA1. It is a half transporter of $75.6 \mathrm{kDa}$ that had been implicated in the regulation of macrophage cholesterol and phospholipid transport. ABCG1 mediates the transport of cholesterol from cells only to HDL but not to lipid-free apoA-I. In addition, it has been demonstrated to play a role in cholesterol movements and trafficking to the plasma membrane (Kobayashi et al., 2006; Vaughan and Oram, 2005). This protein may play a role in cholesterol redistribution to a cell-surface pool rendering it more accessible to enzymatic oxidation and to removal by HDL particles. Similar to ABCA1, the transcriptional regulation of ABCG1 expression by nuclear receptors, namely liver X receptors and related partners, modulates the cellular lipid and cholesterol efflux, respectively (Velamakanni et al., 2007).

ABCG5 and ABCG8 are also half transporters that must form obligatory heterodimers to become functionally active. They are known to be involved in the biliary and intestinal efflux of cholesterol and phyto- and shellfish sterols (Kusuhara and Sugiyama, 2007). They are co-ordinately regulated through nuclear receptors and other regulatory elements. Mutations in either ABCG5 or ABCG8 cause sitosterolemia, a rare autosomal recessive disorder characterized by accumulation of both plant-derived sterols and animal-derived sterols (cholesterol) in plasma and tissues, leading to the development of xanthomas (Velamakanni et al., 2007). The mRNA transcripts of ABCG5 or ABCG8 have been identified in the bovine mammary gland (Farke et al., 2006), and therefore could be potential cholesterol transporters in this tissue. Their protein expression and functional activity in this organ, however, has yet to be elucidated. 


\section{Albrecht, X. Huang and E.C. Ontsouka}

\section{Other cholesterol transport proteins}

NPC proteins are integral membrane proteins that are expressed in cytosolic late endosomes and lysosomes. They prevent intracellular accumulation of cholesterol derived from the cellular uptake of LDL. These proteins exist in two isoforms, NPC1 and NPC2, that bind cholesterol and mediate its trafficking and delivery from late endosomes and lysosomes to other cellular compartments (Pentchev, 2004). The importance of NPC1 and NPC2 for cholesterol, and more generally lipid homeostasis is evidenced in NPC disease where patients with mutations in NPC1 or NPC2, or both, face neurodegeneration and die in childhood due to cholesterol and glycosphingolipid accumulation in vital organs like brain, liver, and lungs (Vanier and Millat, 2003). NPC1 was shown to be expressed and differentially regulated during the pregnancy-lactation cycle in bovine mammary tissue (Mani et al., 2009).

NPC1L1 is a transmembrane protein that shares high amino acid homology with NPC1 and has a sterol-sensing domain similar to other key genes of cholesterol metabolism (Davies et al., 2000). Investigations performed so far showed a differential distribution of NPC1L1 among various tissues in humans, rat or mouse. It has been described to play a role in mediating cholesterol traffic in the intestine (Altmann et al., 2004) or liver (Tang et al., 2011). However, whether this interesting candidate gene is important for cholesterol transport in the mammary gland is currently not known.

\subsubsection{Diffusion mechanism}

In addition to the active transport mechanisms described above, the cholesterol molecule can be transferred from cell membranes to an acceptor by diffusion (McLean and Phillips, 1981). The diffusion of cholesterol from the phospholipid bilayer to an acceptor, such as HDL particles or serum albumin, occurs in a time scale of hours and might be facilitated by plasma membrane proteins. Despite the lack of decisive studies in human mammary tissue, cholesterol is likely to passively diffuse out of mammary alveolar cells (Figure 8.2, pathway B). In own cholesterol efflux studies using primary mammary epithelial cells we observed that after loading of the cells with $\left[{ }^{3} \mathrm{H}\right]$-cholesterol, the isotope diffuses from the cells into the medium in the absence of cholesterol acceptor proteins such as apoA-1 (unpublished data).

\subsubsection{Milk fat globule secretion as a potential transport mechanism}

The milk lipids are secreted by mammary alveolar epithelial cells as emulsified globules (or lipid droplets) surrounded by a protein-rich polar lipid coat called the MFG membrane (Figure 8.1, pathway II and Figure 8.2, pathway C). The size of MFG ranges from less than $1 \mu \mathrm{m}$ to about $10 \mu \mathrm{m}$ in diameter (Keenan, 2001; Mather and Keenan, 1998). As shown in Figure 8.1 and 8.2, MFG start as small lipid droplets in the ER and migrate towards the apical membrane of the secretory mammary cell as they develop and mature. At the apical region, lipid droplets are progressively enveloped by the apical plasma membrane and pinched off into the alveolar lumen as MFG. The plasma membrane derivative covering MFG contains, among other components, 
predominantly phospholipids, sphingolipids and sterols. It has been demonstrated that approx. $80 \%$ of the total milk cholesterol is found within the membrane surrounding the MFG membrane (Keenan, 2001; Patton and Jensen, 1975). Similarly, the major fraction of phospholipids (approx. $60 \%$ of total milk phospholipids) is also derived from the membrane of MFG. The remaining part of both milk cholesterol and phospholipids are found in skim milk (Keenan, 2001; Patton and Jensen, 1975). The core of MFG consists essentially of triglycerides (Keenan, 2001), which is the predominant milk fat component representing $98 \%$ or more of the total milk fat fraction depending on the species. Recently, a variation of cholesterol content in MFG of different sizes has been described, and it was suggested that the assembly of MFG of different sizes might involve specific biosynthetic routes rather than representing a random process (Mesilati-Stahy et al., 2011).

It is not clear yet which of the three potential mechanisms described above represents the major pathway involved in cholesterol transport in the mammary gland and cholesterol transfer into milk. Considering that cholesterol found in milk is predominantly distributed in the membrane of MFG, and that MFG secretion is a continuous and a priori energy-independent process, this pathway might be crucial for the transfer of cholesterol into milk. Whether the distribution of cholesterol in different milk fractions (in milk fat greater than in skim milk) is indicative for different secretory routes has, to our knowledge, never been specifically studied. Interestingly, ABC transporters including ABCA1 and ABCG1 have also been found in the membrane of MFG (Mani et al., 2011). However, whether these proteins may play a functional role in MFG is currently not known.

\subsection{Comparison of $A B C$ cholesterol transporters in lactating and nonlactating mammary tissues}

\subsection{1 mRNA expression levels of cholesterol transporters}

mRNA expression studies of human ABCA1 showed a high expression level in placenta, liver, lung, adrenal glands and foetal tissues, and comparably low expression levels, among other tissues, in the mammary gland (Langmann et al., 1999). However, if the mammary gland tested in the aforementioned study was in the lactating or nonlactating state was not specified. Similar findings have also been reported by other investigators showing a lower expression level of ABCA1 mRNA in bovine mammary tissues as compared to lung or liver (Farke et al., 2006). The tissue profiling of ABCG5 and ABCG8 revealed that both proteins were expressed at relatively low levels in the bovine mammary gland as compared to the liver and the digestive tract (Viturro et al., 2006).

Regarding the comparative analysis between lactating and nonlactating mammary tissues only very few studies report on this topic (Mani et al., 2009, 2010, 2011). The mRNA expression of $\mathrm{ABC}$ transporters has not been profiled in healthy lactating and nonlactating human mammary tissues probably due to ethical problems with regard to the collection of human breast tissues. In a study testing bovine and mouse mammary tissues (Mani et al., 2010), the mRNA expression of 


\section{Albrecht, X. Huang and E.C. Ontsouka}

ABCA1, ABCG1 and ABCA7 was compared at different physiological and functional stages of the mammary gland. Interestingly the mRNA abundance of ABCA1 was significantly higher in nonlactating than in lactating murine mammary tissues, whereas the abundance of ABCG1 and ABCA7 mRNA transcripts was similar at these two stages. In bovine mammary tissues, however, the mRNA abundance of ABCA1, ABCA7 and AGCG1 was significantly greater in nonlactating than in lactating mammary tissues. Generally, there was a comparable mRNA expression pattern between ABCA1 and ABCG1 in murine and bovine mammary tissues, whereas ABCA7 was markedly different between the two species. Interestingly, the intracellular cholesterol transporter NPC1 showed an inverse expression profile to ABCA1 and ABCG1 during the pregnancylactation cycle in bovine mammary tissues. While ABCA1 was significantly increased during the nonlactating stage, the mRNA expression of NPC1 showed a marked suppression in nonlactating as compared to lactating mammary tissues (Mani et al., 2009).

\subsubsection{Protein localization of cholesterol transporters}

$\mathrm{ABC}$ transporter protein expression and localization in the mammary epithelium is a prerequisite for playing a role in cholesterol exchange between mammary cells and their surroundings. ABCA1 and ABCA3 expression was identified by immunohistochemistry in the epithelium of normal as well as in neoplastic human breast tissues, but also in breast cancer cell lines (Schimanski et al., 2009). It was reported that ABCA3 and ABCA1 were highly expressed in normal mammary gland epithelium. In addition, positive cytoplasmic staining of ABCA1 in breast cancer tissues was demonstrated (Schimanski et al., 2009). However, in general, protein data on the expression and localization of cholesterol transporters in mammary tissues is still scarce. Parallel to the above mentioned mRNA data on ABCA1, ABCA7 and ABCG1 in three different species (Mani et al., 2010) the authors also compared the protein expression and localization patterns of these proteins at different lactational stages (Table 8.3 and Figure 8.3). ABCA1, ABCG1 and ABCA7 proteins were stained in the alveolar and ductal epithelium in lactating human mammary tissues (with highest intensity for ABCA1 compared with ABCA7 and ABCG1). In nonlactating mammary tissue only ABCA1 and ABCA7 were detected by immunohistochemisty (Mani et al., 2010).

On the other hand, ABCG1 was present in mammary adipocytes throughout all stages, whereas ABCA1 and ABCA7 were less consistently expressed in this cell type (Table 8.3). This distribution pattern suggests that ABCG1 is potentially involved in the regulation of cholesterol storage in human mammary adipocytes and/or may play a more prominent role in lactating rather than nonlactating mammary tissue.

It was furthermore demonstrated that the ABCA1-, ABCG1- and ABCA7-positive staining was completely absent in MEC of nonlactating murine mammary tissue while ABCA1-positive mammary adipocytes were detected (Table 8.3, Mani et al., 2010). In lactating murine tissues ABCA1, ABCG1, and ABCA7-positive cells were mainly detected in the glandular epithelium with diffuse cytoplasmic distribution. In addition, positive staining for ABCA1, ABCG1, and ABCA7 was localized in mammary adipocytes of lactating murine mammary tissues (Table 8.3). 
Table 8.3. Immunohistological localization of candidate $A B C$ transporters in various cell types of lactating and nonlactating mammary tissues (adapted from Mani et al. (2010) with permission from the American Physiological Society). ${ }^{1}$

\begin{tabular}{|c|c|c|c|c|c|c|}
\hline \multirow[t]{2}{*}{ Species/protein } & \multicolumn{2}{|c|}{ Nonlactating 1} & \multicolumn{2}{|c|}{ Nonlactating 2} & \multicolumn{2}{|c|}{ Lactation } \\
\hline & MEC & $A D$ & MEC & $A D$ & MEC & $A D$ \\
\hline \multicolumn{7}{|l|}{ Homo sapiens } \\
\hline $\mathrm{ABCA} 1$ & ++ & $+/-$ & ++ & + & +++ & + \\
\hline$A B C G 1$ & - & + & - & + & + & + \\
\hline \multirow[t]{3}{*}{ ABCA7 } & + & - & ++ & $-/+$ & ++ & $-/+$ \\
\hline & \multicolumn{2}{|c|}{ Nonlactating } & \multicolumn{2}{|c|}{ Early lactation } & \multicolumn{2}{|c|}{ Mid lactation } \\
\hline & MEC & $A D$ & MEC & $A D$ & MEC & $A D$ \\
\hline \multicolumn{7}{|l|}{ Mus musculus } \\
\hline $\mathrm{ABCA} 1$ & - & +++ & ++ & +++ & ++ & +++ \\
\hline ABCG 1 & - & - & + & ++ & ++ & $+/-$ \\
\hline \multirow[t]{3}{*}{$\mathrm{ABCA7}$} & - & - & ++ & +++ & + & + \\
\hline & \multicolumn{2}{|c|}{ Nonlactating } & \multicolumn{2}{|c|}{ Early lactation } & \multicolumn{2}{|c|}{ Mid lactation } \\
\hline & MEC & SC & MEC & SC & MEC & SC \\
\hline \multicolumn{7}{|l|}{ Bos taurus } \\
\hline $\mathrm{ABCA} 1$ & +++ & + & +++ & - & +++ & + \\
\hline ABCG 1 & + & - & ++ & - & ++ & - \\
\hline
\end{tabular}

1 Estimated expression grade for different cell types is indicated as ' - ', absent; ' $-/{ }^{\prime}$, weak staining intensity; and ' + ' to ' +++ ', low- to high-staining intensity. MEC = mammary secretory epithelial cells; $A D=$ mammary adipocytes; SC = stromal cells. Nonlactating 1 = ante-menopausal; Nonlactating 2 = post-menopausal.

This localization pattern may suggest that these ABC transporters could be involved in lipid secretion and storage by MEC and adipocytes, respectively.

In contrast to the expression pattern in mouse mammary tissues, ABCA1 and ABCG1 -positive cells were identified in the mammary epithelium of both nonlactating and lactating bovine mammary tissues (Table 8.3 and Figure 8.3, adapted from Mani et al., 2010). These cholesterol transporters were localized in the cytoplasm and more frequently in the plasma membrane of MEC often with apical accentuation. Furthermore, ABCA1 was localized in endothelial cells. 


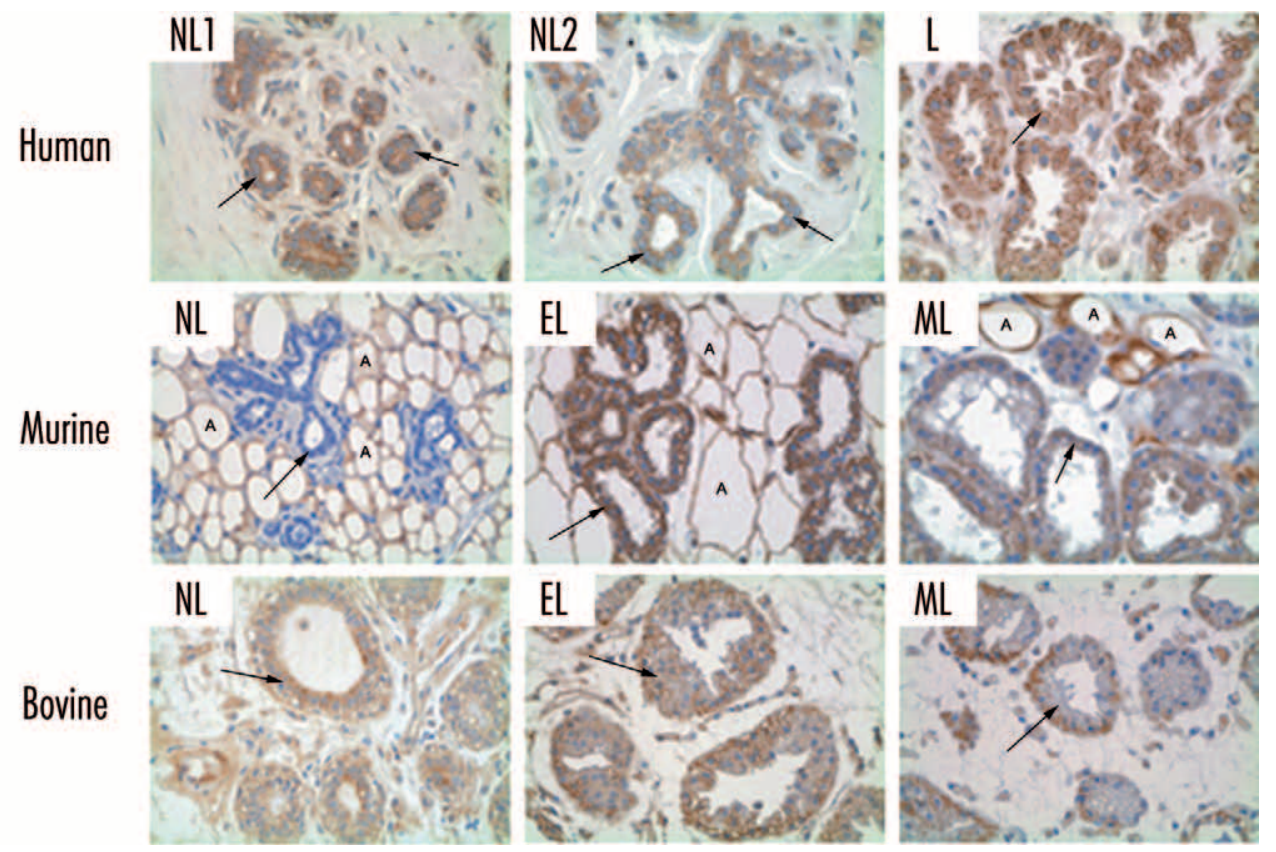

Figure 8.3. Immunohistological localization of the cholesterol transporter ABCA1 in nonlactating and lactating mammary tissues of different species. Mammary gland sections of human, murine and bovine origin were stained with a specific antibody against $A B C A 1$ (brown) and counterstained with hematoxylin (blue). In human breast samples NL1 and NL2 indicate the nonlactating antemenopausal and postmenopausal state, respectively, and L the lactating state. In murine and bovine samples: NL = nonlactating; $E L=$ early lactation (mouse: $1-6$ days after parturition; cow: day 14 of lactation); $M L=$ midlactation (mouse: 9-12 days after parturition; cow: day 88 of lactation). $\mathrm{A}=$ adipocyte. Black arrows indicate mammary epithelial cells. Magnification: $\times 40$ (adapted from Mani et al., 2010 with permission from the American Physiological Society).

The presence of $A B C A 1$ and $A B C G 1$ in murine and bovine mammary tissues supports the assumption that $\mathrm{ABCA1}$ and ABCG1 play a role in lipid transfer by the mammary epithelium.

The data shown in Figure 8.3 and those published by others (Schimanski et al., 2009) suggest a physiological role for ABCA1 in the lactating mammary gland. Furthermore they may suggest that ABCG1 could be more important for processes occurring in mammary secretory cells of lactating rather than nonlactating mammary tissue. Despite the lack of a functional proof so far, the identification and cellular localization of ABCA1 and ABCG1 in human, murine, and bovine mammary tissues suggests that these active cholesterol transporters might contribute to lipid exchanges between mammary alveolar epithelial cells and their surroundings. 


\subsection{Summary, conclusions and perspectives}

Cholesterol is required for normal functioning of the mammary tissue and is released into milk as one of its constituents. Cholesterol is important in MFG formation and is used to substitute the removed apical plasma membrane during the exit of MFG. Milk cholesterol is found mainly in the membrane of the MFG but is also present in skim milk. Cholesterol intake by babies/ infants who consume exclusively whole breast milk in early postnatal life seems not to have negative effects for their immediate and later life in contrast to the well-established detrimental cardiovascular consequences associated with high cholesterol intake at older age. In this context, further elucidation of mechanisms regulating the transport of cholesterol in mammary tissue is of great importance. This might help to design strategies for the optimization of milk cholesterol content that should match both elevated cholesterol requirements in infants during the early postnatal life and the necessity to prevent cardiovascular disease associated with high cholesterol levels in all other milk consumers.

The current scientific evidence suggests that cholesterol transport in the mammary tissue is a bi-directional process in terms of exchange at the basal side facing the blood circulation. This exchange might involve active transporters such as ABCA1. On the other hand, the transfer of cholesterol into milk is mediated probably by both non-energy consuming, ATP-independent MFG secretion and ATP-dependent transport mechanisms involving ABC transporters or potentially other yet unidentified transporters. The implication of active cholesterol transport as one mechanism regulating cholesterol transfer into milk has been predicted by proteomic analysis of the membrane of MFG prepared from human milk which identified the presence of $\mathrm{ABC}$ transporters and associated proteins, such as the cholesterol acceptor apoA-1. The role of $\mathrm{ABC}$ transporter-mediated cholesterol transfer involving ABCA1 and ABCG1 is supported by the localization of the corresponding proteins on the mammary secretory epithelium in lactating and nonlactating human mammary tissues, respectively, as well as in surrounding mammary adipocytes. Very recently, a cell culture model of mammary epithelial cells grown on monolayers in a Transwell ${ }^{\oplus}$ system gave first indications that unstimulated primary mammary epithelial cells are able to efflux $\left[{ }^{3} \mathrm{H}\right]$-labelled cholesterol to apo-A 1 at both the apical and the basal side. Thereby, the extent of cholesterol efflux to the basal side was higher than to the apical direction (Ontsouka et al., 2013). However, it still remains to be elucidated if (1) mammary alveolar epithelial cells transfer cholesterol predominantly via energy-dependent or energy-independent mechanisms; (2) whether these processes are dependent of the physiological and functional stage of the mammary gland; and (3) how hormones specific for the pregnancy-lactation cycle affect these processes.

\section{Acknowledgements}

The present paper was supported by the Swiss National Science Foundation through the National Centre of Competence in Research TransCure. The authors thank Thomas Stübi for his contribution in discussion and literature search during the preparation of the present review. 


\section{Albrecht, X. Huang and E.C. Ontsouka}

\section{References}

Altmann, S.W., Davis, H.R., Jr., Zhu, L.J., Yao, X., Hoos, L.M., Tetzloff, G., Iyer, S.P., Maguire, M., Golovko, A., Zeng, M., Wang, L., Murgolo, N. and Graziano, M.P., 2004. Niemann-Pick C1 Like 1 protein is critical for intestinal cholesterol absorption. Science 303, 1201-1204.

Davies, J. P., Levy, B. and Ioannou, Y.A., 2000. Evidence for a Niemann-pick C (NPC) gene family: identification and characterization of NPC1L1. Genomics 65, 137-145.

Farke, C., Viturro, E., Meyer, H.H. and Albrecht, C., 2006. Identification of the bovine cholesterol efflux regulatory protein ABCA1 and its expression in various tissues. Journal of Animal Science 84, 2887-2894.

Gofflot, F., Hars, C., Illien, F., Chevy, F., Wolf, C., Picard, J.J. and Roux, C., 2003. Molecular mechanisms underlying limb anomalies associated with cholesterol deficiency during gestation: implications of Hedgehog signaling. Human Molecular Genetics 12, 1187-1198.

Isomura, H., Takimoto, H., Miura, F., Kitazawa, S., Takeuchi, T., Itabashi, K. and Kato, N., 2011. Type of milk feeding affects hematological parameters and serum lipid profile in Japanese infants. Pediatrics International $53,807-813$.

Jandal, J.M., 1996. Comparative aspects of goat and sheep milk. Small Ruminant Research 22, 177-185.

Jensen, R.G., 1996. The lipids in human milk. Progress in Lipid Research 35, 53-92.

Jensen, R.G., 2002. The composition of bovine milk lipids: January 1995 to December 2000. Journal of Dairy Science $85,295-350$.

Keenan, TW., 2001. Milk lipid globules and their surrounding membrane: a brief history and perspectives for future research. Journal of Mammary Gland Biology and Neoplasia 6, 365-371.

Kobayashi, A., Tanezawa, Y., Hirata, T., Shimizu, Y., Misasa, K., Kioka, N., Arai, H., Ueda, K. and Matsuo, M., 2006. Efflux of sphingomyelin, cholesterol, and phosphatidylcholine by ABCG1. Journal of Lipid Research 47, 1791-1802.

Kusuhara, H. and Sugiyama, Y., 2007. ATP-binding cassette, subfamily G (ABCG family). Pflugers Archiv European Journal of Physiology 453, 735-744.

Langmann, T., Klucken, J., Reil, M., Liebisch, G., Luciani, M. F., Chimini, G., Kaminski, W.E. and Schmitz, G., 1999. Molecular cloning of the human ATP-binding cassette transporter 1 (hABC1): evidence for sterol-dependent regulation in macrophages. Biochemical and Biophysical Research Communications 257, 29-33.

Lee, J., Shirk, A., Oram, J.F., Lee, S.P. and Kuver, R., 2002. Polarized cholesterol and phospholipid efflux in cultured gall-bladder epithelial cells: evidence for an ABCA1-mediated pathway. Biochemical Journal 364, 475-484.

Linsel-Nitschke, P., Jehle, A. W., Shan, J., Cao, G., Bacic, D., Lan, D., Wang, N. and Tall, A.R., 2005. Potential role of ABCA7 in cellular lipid efflux to apoA-I. Journal of Lipid Research 46, 86-92.

Liu, Y. and Tang, C., 2012. Regulation of ABCA1 functions by signaling pathways. Biochimica et Biophysica Acta 1821, 522-529.

Mani, O., Korner, M., Ontsouka, C.E., Sorensen, M. T., Sejrsen, K., Bruckmaier, R.M. and Albrecht, C., 2011. Identification of ABCA1 and ABCG1 in milk fat globules and mammary cells - implications for milk cholesterol secretion. Journal of Dairy Science 94, 1265-1276.

Mani, O., Korner, M., Sorensen, M.T., Sejrsen, K., Wotzkow, C., Ontsouka, C.E., Friis, R.R., Bruckmaier, R.M. and Albrecht, C., 2010. Expression, localization, and functional model of cholesterol transporters in lactating and nonlactating mammary tissues of murine, bovine, and human origin. American Journal of Physiologyregulatory Integrative and Comparative Physiology 299, R642-654. 


\section{Cholesterol transporters in human mammary tissue}

Mani, O., Sorensen, M.T., Sejrsen, K., Bruckmaier, R.M. and Albrecht, C., 2009. Differential expression and localization of lipid transporters in the bovine mammary gland during the pregnancy-lactation cycle. Journal of Dairy Science 92, 3744-3756.

Mather, I.H. and Keenan, T.W., 1998. Origin and secretion of milk lipids. Journal of Mammary Gland Biology and Neoplasia 3, 259-273.

McLean, L.R. and Phillips, M.C., 1981. Mechanism of cholesterol and phosphatidylcholine exchange or transfer between unilamellar vesicles. Biochemistry 20, 2893-2900.

Mesilati-Stahy, R., Mida, K. and Argov-Argaman, N., 2011. Size-dependent lipid content of bovine milk fat globule and membrane phospholipids. Journal of Agricultural and Food Chemistry 59, 7427-7435.

Mishkel, M.A., 1974. Neonatal plasma lipids as measured in cord blood. Canadian Medical Association Journal $111,775-780$.

Monks, J., Huey, P.U., Hanson, L., Eckel, R.H., Neville, M.C. and Gavigan, S., 2001. A lipoprotein-containing particle is transferred from the serum across the mammary epithelium into the milk of lactating mice. Journal of Lipid Research 42, 686-696.

Nagao, K., Takahashi, K., Azuma, Y., Takada, M., Kimura, Y., Matsuo, M., Kioka, N. and Ueda, K., 2012. ATP hydrolysis-dependent conformational changes in the extracellular domain of ABCA1 are associated with apoA-I binding. Journal of Lipid Research 53, 126-136.

Ontsouka, C.E., Bruckmaier, R.M. and Blum, J.W., 2003. Fractionized milk composition during removal of colostrum and mature milk. Journal of Dairy Science 86, 2005-2011.

Ontsouka, E.C., Huang, X., Stieger, B., Albrecht, C., 2013. Characteristics and functional relevance of apolipoprotein-A1 and cholesterol binding in mammary gland tissues and epithelial cells. PLoS ONE 8(7): e70407.

Owen, C.G., Martin, R.M., Whincup, P.H., Smith, G.D. and Cook, D.G., 2005. Effect of infant feeding on the risk of obesity across the life course: a quantitative review of published evidence. Pediatrics 115, 1367-1377.

Park, Y.W., Juarez, M., Ramos, M. and Haenlein, G.F.W., 2007. Physico-chemical characteristics of goat and sheep milk. Small Ruminant Research 68, 88-113.

Patton, S. and Jensen, R.G., 1975. Lipid metabolism and membrane functions of the mammary gland. Progress in the Chemistry of Fats and other Lipids 14, 163-277.

Pentchev, P.G., 2004. Niemann-Pick C research from mouse to gene. Biochimica et Biophysica Acta 1685, 3-7.

Picaud, J.C., Chapalain, V., Paineau, D., Zourabichvili, O., Bornet, F.R. and Duhamel, J.F., 2010. Incidence of infectious diseases in infants fed follow-on formula containing synbiotics: an observational study. Acta Paediatrica 99, 1695-1700.

Picciano, M.F., 2001. Representative values for constituents of human milk. Pediatric Clinics of North America 48, 263-264.

Reinhardt, T.A. and Lippolis, J.D., 2006. Bovine milk fat globule membrane proteome. Journal of Dairy Research 73, 406-416.

Rudnicka, A.R., Owen, C.G. and Strachan, D.P., 2007. The effect of breastfeeding on cardiorespiratory risk factors in adult life. Pediatrics 119, e1107-1115.

Schimanski, S., Wild, P.J., Treeck, O., Horn, F., Sigruener, A., Rudolph, C., Blaszyk, H., Klinkhammer-Schalke, M., Ortmann, O., Hartmann, A. and Schmitz, G., 2009. Expression of the lipid transporters ABCA3 and ABCA1 is diminished in human breast cancer tissue. Hormone and Metabolic Research 42, 102-109. 


\section{Albrecht, X. Huang and E.C. Ontsouka}

Stefulj, J., Panzenboeck, U., Becker, T., Hirschmugl, B., Schweinzer, C., Lang, I., Marsche, G., Sadjak, A., Lang, U., Desoye, G. and Wadsack, C., 2009. Human endothelial cells of the placental barrier efficiently deliver cholesterol to the fetal circulation via ABCA1 and ABCG1. Circulation Research 104, 600-608.

Takahashi, K., Kimura, Y., Nagata, K., Yamamoto, A., Matsuo, M. and Ueda, K., 2005. ABC proteins: key molecules for lipid homeostasis. Medical Molecular Morphology 38, 2-12.

Tang, W., Jia, L., Ma, Y., Xie, P., Haywood, J., Dawson, P. A., Li, J. and Yu, L., 2011. Ezetimibe restores biliary cholesterol excretion in mice expressing Niemann-Pick C1-Like 1 only in liver. Biochimica et Biophysica Acta 1811, 549-555.

Ueda, K., 2011. ABC proteins protect the human body and maintain optimal health. Bioscience Biotechnology and Biochemistry 75, 401-409.

Vanier, M. T. and Millat, G., 2003. Niemann-Pick disease type C. Clinical Genetics 64, 269-281.

Vaughan, A.M. and Oram, J.F., 2005. ABCG1 redistributes cell cholesterol to domains removable by high density lipoprotein but not by lipid-depleted apolipoproteins. The Journal of Biological Chemistry 280, 30150-30157.

Velamakanni, S., Wei, S., Janvilisri, T. and Van Veen, H., 2007. ABCG transporters: structure, substrate specificities and physiological roles: a brief overview. Journal of Bioenergetics and Biomembranes 39, 465-471.

Victora, C.G., Vaughan, J.P., Lombardi, C., Fuchs, S.M.C., Gigante, L.P., Smith, P.G., Nobre, L.C., Teixeira, A. M.B., Moreira, L.B. and Barros, F.C., 1987. Evidence for protection by breast-feeding against infant deaths from infectious-diseases in Brazil. Lancet 355, 319-322.

Viturro, E., Farke, C., Meyer, H.H. and Albrecht, C., 2006. Identification, sequence analysis and mRNA tissue distribution of the bovine sterol transporters ABCG5 and ABCG8. Journal of Dairy Science 89, 553-561.

Viturro, E., Koenning, M., Kroemer, A., Schlamberger, G., Wiedemann, S., Kaske, M. and Meyer, H.H., 2009. Cholesterol synthesis in the lactating cow: induced expression of candidate genes. Journal of Steroid Biochemistry and Molecular Biology 115, 62-67.

Wang, N., Lan, D., Gerbod-Giannone, M., Linsel-Nitschke, P., Jehle, A.W., Chen, W., Martinez, L.O. and Tall, A.R., 2003. ATP-binding cassette transporter A7 (ABCA7) binds apolipoprotein A-I and mediates cellular phospholipid but not cholesterol efflux. Journal of Biological Chemistry 278, 42906-42912.

Wang, Y., Tong, J., Li, S., Zhang, R., Chen, L., Zheng, M., Wang, M., Liu, G., Dai, Y., Zhao, Y. and Li, N., 2011. Overexpression of human lipoprotein lipase in mouse mammary glands leads to reduction of milk triglyceride and delayed growth of suckling pups. PLoS One 6, e20895. 